\title{
Social Recommendation Model Regularized with User Trust and Item Ratings
}

\author{
Dr. G. Anandharaj ${ }^{1}$, M. Anu ${ }^{2}$ \\ ${ }^{1}$ AssociateProfessor \& Head, Department of Computer Science and Applications, Adhiparasakthi College of Arts and Science \\ (Autonomous), Kalavai, Vellore - 632506, India
}

${ }^{2}$ Research Scholar, Department of Computer Science and Applications, Adhiparasakthi College of Arts and Science (Autonomous), Kalavai, Vellore - 632506, India

\begin{abstract}
Social trust aware recommender systems have been well studied in recent years. However, most of existing methods focus on the recommendation scenarios where users can provide explicit feedback to items. But in most cases, the feedback is not explicit but implicit. Moreover, most of trust aware methods assume the trust relationships among users are single and homogeneous, whereas trust as a social concept is intrinsically multi-faceted and heterogeneous. Simply exploiting the raw values of trust relations cannot get satisfactory results. Based on the above observations, we propose to learn a trust aware personalized ranking method with multi-faceted trust relations for implicit feedback. Recommender systems have been widely used to provide users with high-quality personalized recommendations from a large volume of choices. Robust and accurate recommendations are important in e-commerce operations (e.g., navigating product offerings, personalization, improving customer satisfaction), and in marketing (e.g., tailored advertising, segmentation, cross-selling). The former issue refers to the fact that users usually rate only a small portion of items while the latter indicates that new users only give a few ratings (a.k.a. cold-start users). Both issues severely degrade the efficiency of a recommender system in modeling user preferences and thus the accuracy of predicting a user's rating for an unknown item.
\end{abstract}

Keywords: Social trust, implicit ratings, explicit ratings, e-commerce

\section{Introduction}

With the exponential growth of information generated on the World Wide Web, recommender systems as one of the efficient information filtering techniques have attracted a lot of attentions in the last decade. Recommender systems focus on solving the information overload problem by suggesting the items that are potential of their interests to users. Typical recommender systems are based on collaborative filtering, which is a technique that can predict the preference of a given user by only collecting rating information from other similar users or items[1-2]. Examples of successful applications of recommender systems can be found in many industries, such as movie recommendation at Netflix and product recommendation at Amazon.

However, traditional recommender systems only utilize the user-item rating matrix for recommendation, and ignore the social connections or trust relations among users. But in our real life, we always turn to our friends we trust for recommendations of products, consultations, music and movies. The social trust relation helps us locate the items we are potentially interested in. Hence, with the advent of online social networks, social trust aware recommender systems have drawn lots of attentions. For example, Ma et al.explored several ways to incorporate trust relations into the matrix factorization framework. Noel et al. improved the existing social matrix factorization objective functions, and proposed a new unified framework for social recommendation.

Unfortunately, most of these existing trust aware recommendation methods are proposed for social networks with explicit feedback of users. In these cases, a user can tell us to what extent he/she likes a specific item by giving a real-valued rating, and we can explicitly know what he/she likes and hates. Nevertheless, explicit feedback is not always available. Most of the feedback in real social networks is not explicit but implicit. In implicit feedback social networks, we can only get a user's positive behaviors from the history of what he/she has clicked, purchased or connected, but never know to what degree he/she likes and what he/she does not like. The learning task for this kind of data is how to infer the user preferences from only positive observations. Rendle et al. explored this problem in, where they made use of partial order of items and presented ageneric Bayesian optimization criterion for personalized ranking. Their work provides us a general way to learn users' interests from implicit data. However they didnot consider the impact of social trust relations, which have been demonstrated in the rating prediction based tasks.

Moreover, most of the existing social trust aware recommendation methods assume the trust relationships among users are single and homogeneous. For example, Jamali and Ester incorporated the mechanism of trust propagation into a matrix factorization method, and the binary social relations were considered. However, trust as a social concept is intrinsically multi-faceted and heterogeneous. Simply exploiting the raw values of trust relations cannot get satisfactory results. Intuitively, a user may trust different people indifferent domains/categories. For example, in multi category recommender systems, a user may trust an expert in movies category but not trust him/her in cars category. Treating trust relationships of different categories equally will not capture the multi-faceted features hidden below the surface (especially when the social relations only have binary values) 


\section{International Journal of Science and Research (IJSR)}

ISSN (Online): 2319-7064

Index Copernicus Value (2015): 78.96 | Impact Factor (2015): 6.391

\section{Related Work}

\subsection{A Trust-based recommendation model}

In social rating networks a user can label (add) other users as trusted friends and thus form a social network. Trust is not symmetric; for example, users $u_{1}$ trusts $u_{a}$ but $u_{a}$ does not specify user $u_{1}$ as trustworthy. Besides, users can rate aset of items using a number of rating values, e.g., integers from 1 to 5. These items could be products, movies, music,etc. of interest. The recommendation problem in this work is to predict the rating that a user will give to an unknown item, for example, the value that user $u_{a}$ will give to item $i_{\mathfrak{a}}$, based on both a user-item rating matrix and a user-user trust matrix. Other well-recognized recommendation problems include for example top- $\mathrm{N}$ item recommendation.

Suppose that a recommender system includes $m$ users and $n$ items. Let $R=\left[r_{u_{i} i}\right]_{m \times n}$ denote the user-item rating matrix, where each entry $r_{\mathrm{L}_{S} \mathrm{i}}$ represents the rating given by user $u$ on item. For clarity, we preserve symbols $u, v$ for users, and $i_{i} j$ for items. Since a user only rated a small portion of items, the rating matrix $\mathrm{R}$ is only rated a small portion of items, the rating matrix $\mathrm{R}$ is only partially observed and oftentimes very sparse. Let $I_{u}=\left\{i \mid r_{u_{L} i} \neq 0\right\}$ denote the set of items rated by user $u$. Let $p_{u}$ and $q_{i}$ be a ddimensional latent feature vector of user $u$ and item, respectively. The essence of matrix factorization is to find two low-rank matrices: user-feature matrix $P \in \mathbb{R}^{d \times m}$ and item-feature matrix $Q \in \mathbb{R}^{d x n}$ that can adequately recover the rating matrix R, i.e., $\approx P^{T} Q$, where $P^{T}$ is the transpose of matrix $P$. The underlying assumption is that both users and items can be characterized by a small number of features. Hence, the rating on item $j$ for user $u$ can be predicted by the inner product of user-specific vector $p_{u}$ and item-specific vector $q_{j}$ i.e., $\hat{r}_{u_{j} j}=q_{j}^{T} P_{u}$. In this regard, the main task of recommendations is to predict the rating $\hat{r}_{\mathrm{u}_{j} j}$ as close as possible to the ground truth $r_{\mathrm{u}_{0} j}$. Formally, we can learn the user- and item-feature matrices by minimizing the following loss (objective ) function:

$\mathcal{L}_{\mathrm{Y}}=\frac{1}{2} \sum_{\mathrm{u}} \sum_{j \in I_{u}}\left(q_{j}^{T} p_{u}-r_{u_{j} j}\right)^{2}+\frac{\lambda}{2}\left(\Sigma_{\mathrm{u}}\left\|p_{u}\right\|_{F}^{2}+\Sigma_{j}\left\|q_{u}\right\|_{F}^{2}\right)$,

Where $\left\|_{s}\right\|_{F}$ denotes the Frobenius norm, and $\lambda$ is a parameter to control model complexity and to avoid overfitting.

On the other hand, suppose that a social network is represented by a graph $\mathcal{G}=(\nu, \varepsilon)$, where $\nu$ includes a set of $\mathrm{m}$ nodes (users) and $\varepsilon$ represents the directed trust relationships among users. We can use the adjacency matrix $T=\left[t_{\text {uv }}\right]_{\text {mxm }}$ to describe the structure of edges $\varepsilon$, where $t_{u_{v}}$ indicates the extent to which users $u$ trusts $v$. Usually, only binary values are used, i.e, $t_{u v}=1$ means that user $u$ trusts user $v$ whereas $t_{u_{v}}=0$ indicates the non-trust relationship. Similarly, the trust matrix $\mathrm{T}$ is also very sparse. We denote $p_{u}$ and $w_{u}$ as the $d-$ dimensional lantent feature vector of truster $u$ and trustee , respectively. We limit the trusters in the trust matrix and the active users in the rating matrix to share the same user-feature space in order to bridge them together. Hence, we have truster-feature matrix $P^{d \times m}$ and trustee-feature matrix $W^{d \times m}$. By employing the low- rank matrix approximation, we can recover the trust matrix by $T \approx P^{T} W$. Thus, a trust relationship can be predicted by the inner product of a truster-specific vector and a trustee-specific vector $\hat{t}_{\mathrm{u}_{j} j}=w_{j}^{T} P_{\mathrm{u}}$. The matrices $\mathrm{P}$ and $\mathrm{W}$ can be learned by minimizing the following loss function:

$\mathcal{L}_{y}=\frac{1}{2} \sum_{u} \sum_{j \in T_{u}^{+}}\left(w_{j}^{T} p_{u}-t_{u_{u} j}\right)^{2}+\frac{\lambda}{2}\left(\sum_{u}\left\|p_{u}\right\|_{F}^{2}+\sum_{j}\left\|w_{u}\right\|_{F}^{2}\right.$

Where $T_{u}^{+}$is the set of users trusted by user $u$, i.e., the set of out-going trusted users.

\subsection{Implicit Influence of Trusted Users}

The trusted users of an active user have an effect on rating prediction for a certain item. We take into account this effect by modelling user preference in the same manner as rated items, given by:

$$
\hat{r}_{u_{j} j}=b_{u, j}+q_{j}^{T}\left(p_{u}+\left|I_{u}\right|^{-\frac{1}{2}} \sum_{i \in I_{u}} y_{i}+\|\left. T_{u}^{+}\right|^{-\frac{1}{2}} \Sigma_{v \in T_{u}^{+}} w_{v}\right)
$$

Where $\quad b_{u_{j} j}=b_{u}+b_{j}+\mu$ hereafter represents bias terms, $w_{u}$ is the user-specific latent feature vector of users (trustees) trusted by user $u_{v}$ and thus $q_{j}^{T} w_{v}$ can be explained by the ratings predicted by the trusted users, i.e., the influence of trustees on the rating prediction. In other words, the inner product $q_{j}^{T} w_{v}$ indicates how trusted users influence user $u^{r}$ s rating on item $j$. Similar to ratings, a user's feature vector can be interpreted by the set of users whom she trust, i.e., $\left|T_{u}^{+}\right|^{-\frac{1}{2}} \sum_{v \in T_{u}^{+}} w_{V}$. Therefore, a user $u$, is further modeled by $\left(p_{u}+\left|I_{u}\right|^{-\frac{1}{2}} \sum_{i \in I_{u}} y_{i}+\left|T_{u}^{+}\right|^{-\frac{1}{2}} \sum_{v \in T_{u}^{+}} w_{v}\right)$ in the social rating networks, considering the influence of both rated items and trusted users.

\subsection{Implicit Influence of Trusting Users.}

The trusting users of an active user can also influence the rating prediction for a certain item. In fact, Observation has indicated that such influence may be comparable with that of trusted users. Similarly, the effect can be considered by modelling user preference, given by:

$$
\hat{r}_{u, j}=b_{u, j}+q_{j}^{T}\left(p_{u}+\left|I_{u}\right|^{-\frac{1}{2}} \sum_{i \in I_{u}} y_{i}+\left|T_{u}^{-}\right|^{-\frac{1}{2}} \sum_{v \in T_{u}^{-}} p_{k}\right)
$$




\section{International Journal of Science and Research (IJSR) \\ ISSN (Online): 2319-7064}

Index Copernicus Value (2015): 78.96 | Impact Factor (2015): 6.391

Where $T_{u}^{-}$is the set of users who trust user $u$, i.e., theset of her trusters. Thus, $q_{i} p_{k}$ can be explained by theratings predicted by the trusting users, i.e., the influence of trusters on the predictions. Similarly, the inner product $q_{j}^{T} p_{k}$ indicates how trusting users $k$ influence user $u$ 's ratingon item j. Similar to ratings, a user's feature vector can be interpreted by the set of users whom trust her, i.e., $\left|T_{u}^{-}\right|^{-\frac{1}{2}} \Sigma_{\text {VE T }}-p_{k}$. Therefore, a user $u$ is further modeled $\left(p_{u}+\left|I_{u}\right|^{-\frac{1}{2}} \sum_{i \in I_{u}} y_{i}+\left|T_{u}^{-}\right|^{-\frac{1}{2}} \sum_{v \in T_{u}^{-}} p_{k}\right)$ in the social

rating networks, considering the influence of both rated items and trusting users.

\subsection{Combinational Implicit Trust Influence.}

The implicit influence of trust neighbors on rating prediction therefore consists of two parts: the influence of both trustees and trusters. To consider both cases, we propose the following fusion approaches.

Linear Combination. A natural and straight forward way is to linearly combine the two kinds of implicit trust influence, given by:

$$
\hat{r}_{u_{j} j}=b_{u_{j} j}+q_{j}^{T}\left(p_{u}+\left|I_{u}\right|^{-\frac{1}{2}} \sum_{i \in I_{u}} y_{i}+\alpha\left|T_{u}^{-}\right|^{-\frac{1}{2}} \sum_{v \in T_{u}^{-}} w_{v}+(1-\alpha)\left|T_{u}^{-}\right|^{-\frac{1}{2}} \sum_{k \in T_{u}^{-}} p_{k}\right),-
$$

Where $\alpha \epsilon[0,1]$ controls the importance of influence of trustees in rating prediction. Specifically, $\alpha=0$ means that we only consider the influence of trusting users; $\alpha=1$ indicates that only the influence of trusted users are considered' and $\alpha \in(0,1)$ mixes the two kinds of trust influence together. In the case of undirected social relationships (e.g., friendship),$T_{u}^{+}$will be equivalent with $T_{\mathrm{u}}^{-}$- and thus the linear combination ensures that our model can be applied to both trust and turst-alike relationships.

\section{Modules}

\subsection{All as Trusting Users}

In a trust relationship, a user ucan be represented either by $p_{u}$ as trustor or by wu as trustee. An alternative way is to model the influence of user $u$ 'strust neighbors, including both trusted and trusting users, in the manner of trusting users such that we can yield the following function:

$$
\hat{r}_{u_{j} j}=b_{u, j}+q_{j}^{T}\left(p_{u}+\left|I_{u}\right|^{-\frac{1}{2}} \sum_{i \in I_{u}} y_{i}+\left|T_{u}^{-}\right|^{-\frac{1}{2}} \sum_{k \in T_{u}^{-}} p_{k}\right)
$$

Where $T_{u}=T_{u}^{+} \cup T_{u}^{-}$denotes the set of user $u$ 's trust neighbors. The underlying assumption is not to distinguish

$$
\mathcal{L}=\frac{1}{2} \sum_{u} \sum_{j \in I_{u}}\left(\hat{r}_{u, j}-r_{u, j}\right)^{2}+\frac{\lambda_{t}}{2}\left(\sum_{u}\left(\propto \sum_{v \in T_{u}^{+}}\left(\hat{t}_{u_{j} j}-t_{u_{j} j}\right)^{2}+(1-\alpha) \sum_{k \in T_{u}^{-}}\left(\hat{t}_{u_{j} j}-t_{u, j}\right)^{2}\right)\right.
$$

where $\hat{t}_{u, j}=w_{v}^{T} p_{u}$ pu is the predicted trust between users $u$ and $v$ computed by the inner product of truster and trustee vectors, i.e., $w_{v}^{T} p_{u}$. Similarly, $\hat{t}_{u_{j} j}=w_{v}^{T} p_{u}$ is the predicted trust for user $k$ towards user $u$, and $\lambda_{t}$ controls the degree of trust regularization.

Trust Walker, a random walk model that combines an item based ranking method and a trust-based nearest neighbor model. Yuan et al fuse two kinds of social relationships ,i.e., friendship and membership in a unified matrix factorization model. In this article, we only consider one kind of social relationships, i.e., either trust or trust-alike, but weverify the generality and application of our model to both kinds of social relationships. Give a state-of-the-art model known as

\subsection{Item Recommendation}

the roles of trust neighbors, but to treat them uniformly in terms of implicit trust influence.

\subsection{All as Trusted Users.}

With the same assumption, we may model the influence of all trust neighbors in the manner of trusted users. That is, we predict the user's possible rating on a target item by:

Where $T_{u}$ is the set of user $u$ 's trust neighbors.

Explicit Trust Influence. In addition, as explained earlier, we rating matrix and those decomposed from the trust matrix share the same feature space in order to bridge both matrices together. In this way, these two types of information can be exploited in a unified recommendation model. Specifically, we can regularize the user-specific vectors $p_{u}$ by recovering the social relationships with other users. The new objective function (without the other regularization terms) is given by:

$$
\hat{r}_{u_{j} f}=b_{u, j}+q_{j}^{T}\left(p_{u}+\left|I_{u}\right|^{-\frac{1}{2}} \sum_{i \in I_{u}} y_{i}+\left|T_{u}\right|^{-\frac{1}{2}} \Sigma_{v \in T_{u}} w_{v}\right)
$$
constrain that the user-specific vectors decomposed from the

Bayesian personalized ranking (BPR) for item recommendation based on implicit feedback. The basic idea is to assume that a rated item for an active user is preferred to an unrated item. However, negative samples may be due to the unawareness of items rather than dislike.

\subsection{User Item/Review Matrix Factorization}

User rating prediction model, Trust SVD works well by incorporating trust influence. The trusted users of an active user have an effect on rating prediction for a certain item. We take into account this effect by modelling user preference in the same manner as rated items.

The implicit influence of trust neighbors on rating prediction therefore consists of two parts: the influence of both trustees 


\section{International Journal of Science and Research (IJSR) \\ ISSN (Online): 2319-7064}

Index Copernicus Value (2015): 78.96 | Impact Factor (2015): 6.391

and trusters. To consider both cases, we propose the following fusion approaches.

(1) Linear Combination.

(2) All as Trusting Users.

(3) All as Trusted Users.

In Expicit Influence The user-specific vectors decomposed from the rating matrix and those decomposed from the trust matrix share the same feature space in order to bridge both matrices together. In this way, these two types of information can be exploited in a unified recommendation model.

Specifically, we can regularize the user-specific vectors by recovering the social relationships with other users. The new objective function (without the other regularization terms)

\subsection{User Rank/Review Matrix Factorization}

The first method sorts all the items based on their popularity, so that the top recommended items are the most popular one in terms of the number of times bought by users. This simple measure is supposed to have reasonable performance, as people tend to focus on few popular items. The ranking score of an item (both explicitly and implicitly). The ranking order between a rated item and an unrated item (but rated by trust users) may be critical to learn users' ranking patterns. the basic matrix factorization (MF) approach for recommendation using only user-item rating matrix.

BaseMF: This method is the baseline matrix factorization approach. This does not take the social network into account

-STE: This is the model proposed which takes into account the social network in a way different from SocialMF. We set $\alpha=0.4$ for STE is the optimum value according to the results of experiments.

- CF: This is the well-known user based collaborative filtering method which is a memory based approach.

\section{Social Trust Aware Ranking Criteria}

\subsection{Social Trust Assumption}

Suppose we have a directed social trust graph $G=(\mathcal{U}, \varepsilon)_{x}$ where $U$ is the set of nodes and $\varepsilon$ is the set of edges. Each node in $U$ represents a user in the network and each edge in $\mathrm{E}$ represents a trust relation between two users. Let $S=\left(S_{w v}\right)_{m \times m}$ denote the $m \times m$ social trust matrix of graph G.

BPRMF makes an assumption, that is, for any two users $u$ and $\mathrm{v}$, the pair-wise preference of user $\mathrm{u}$ is independent of that of user v. But in real world, we always turn to our friends we trust for recommendations. The behavior of a user $u$ is affected by his/her direct neighbors $N_{u}$. Useru's taste is close to those of the neighbors that he/she trusts. In other words, the latent feature vector of $\mathrm{u}$ is close to the latent feature vectors of all his/her trusted neighbors $\in N_{\mathcal{u}}$.

$$
\widehat{U}_{u}=\frac{\sum_{v \in \mathbb{N}_{u} s_{u v} U_{u}}}{\sum v \in \mathbb{N}_{u} s_{u v}}=\sum_{v \in N v} s_{u v}^{*} U_{v}
$$

Where $\widetilde{U_{u}}$ is the estimated latent feature vector of $u_{x} s_{w v}$ is the trust value of how much user $\mathrm{u}$ trusts user v. In most cases, the statement of trust relations only takes on positive values $\left(s_{W V}=1\right)$, which will not fully reflect the true relationship between two users.

The user latent feature vector $U$ now has two factors: the zero-mean spherical Gaussian prior, and the conditional distribution of $U$ given the latent features of his/her trusted neighbors. Hence,

$$
\begin{aligned}
& p\left(U \mid S_{v} \sigma_{U}^{2}, \sigma_{S}^{2}\right) \propto p\left(U \mid \sigma_{U}^{2}\right) p\left(U \mid S_{s} \sigma_{s}^{2}\right) \\
& =\prod_{u=1}^{m} \mathcal{N}\left(U_{u} \mid o_{v} \sigma_{U}^{2} I\right) \times \prod_{u=1}^{m} \mathcal{N}\left(U_{u} \mid \sum_{t \in N_{u}} s_{u v}^{*} U_{t}, \sigma_{S}^{2} I\right)
\end{aligned}
$$

\subsection{Learning the Strengths of Multi-Faceted Trust Relations}

Social BPR assumes the social trusts in different categories have the same influence on the target user and the trust relations only take on values from either 1 or 0 , which do not truly reflect their impact on user behaviors. However, in real-world social networks, trust is multi-faceted, that is, a user places different trusts in different categories. Hence, it is desirable to understand the true trust strengths from multicategory systems. In multi-category systems, each user can have multiple favorite categories, and will make social trusts based on his/her category interests.

Given the social trust network and category similarity, we propose a Category-Sensitive Random Walk with Restart (CRWR) method to learn the intrinsic relevance between two users. For a standard random walk with restart approach, a random walker starts from the i-th vertex, iteratively with probability $1-\alpha$ jumps to other vertices according to transition probabilities $q_{i}=\left\{q_{i 1}, \ldots, q_{i n}\right\} x$ and with probability ajumps back to itself. After reaching the steady state, the probability of the random walker staying at the $\mathrm{j}$-th vertex corresponds to the relevance score of vertex I to vertex $\mathrm{j}$.

To reflect users' multi-category interests, we bias the transition matrix $\mathbf{Q}$ by users' category similarity matrix $\mathbf{W}$ which can be defined as follows:

$$
Q=D^{-1} \cdot W_{x}
$$

where $\mathrm{D}$ is the degree matrix of trust graph G. In this scene, when the random walker jumps to other vertices, the walker will not only consider the graph structure but also consider the category similarity between two users. The final steadystate probability matrix can be obtained by iterating the following updates:

$$
B(t+1)=(1-\infty) Q B(t)+\infty I_{x}
$$

where $B(t)$ and $B(t+1)$ are the state probability matrices at time $t$ and time $t+1$ respectively. The iterations will finally converge when $t \rightarrow \infty$. For any two vertexes $i$ and $j$, the value of steady state probability $b_{\text {ij }}$ represents how well user $i$ trusts/knows user $j$ in social graph.

\subsection{Architecture}


International Journal of Science and Research (IJSR)

ISSN (Online): 2319-7064

Index Copernicus Value (2015): 78.96 | Impact Factor (2015): 6.391
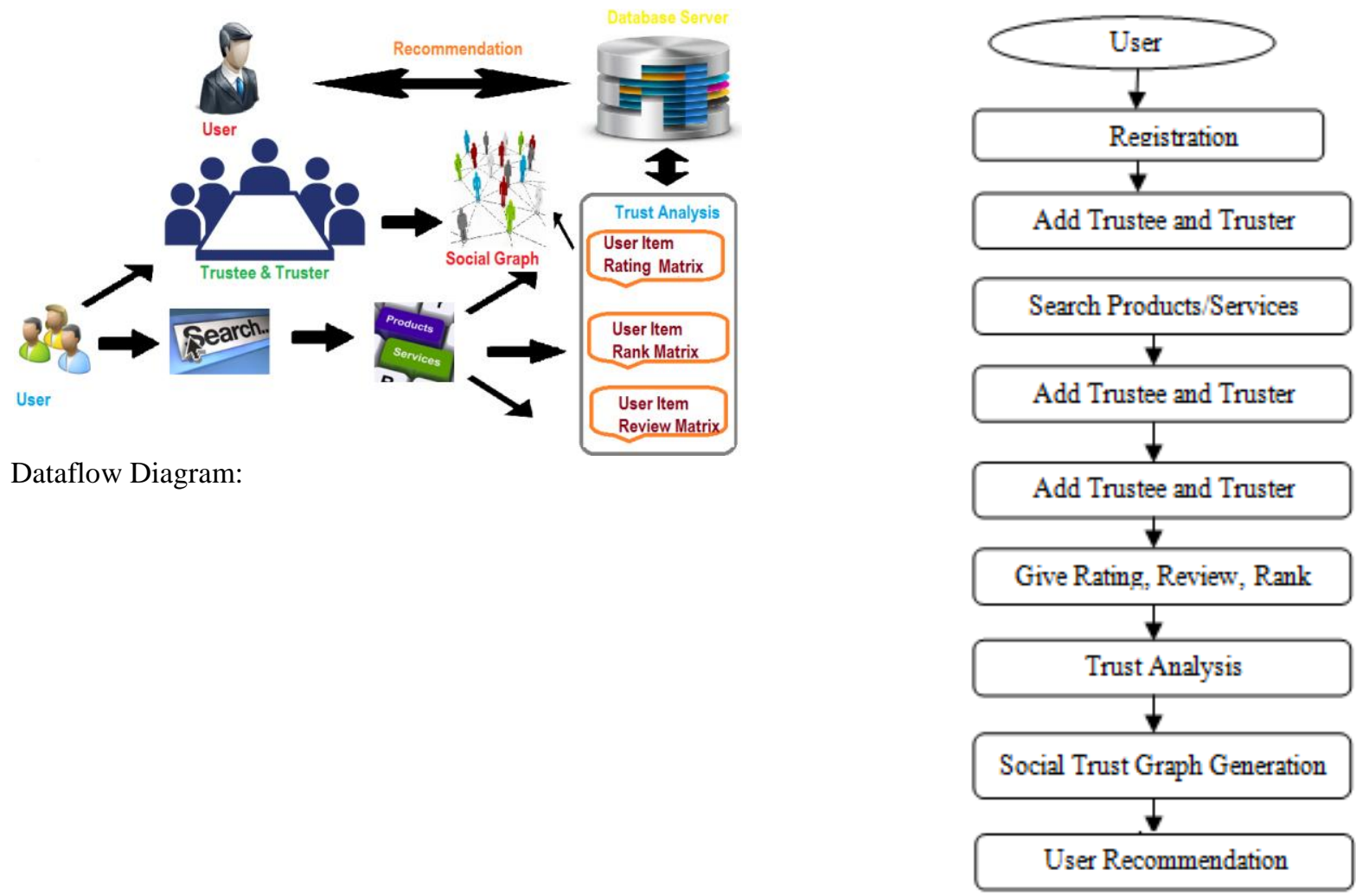

\section{Sample Screen Shots}

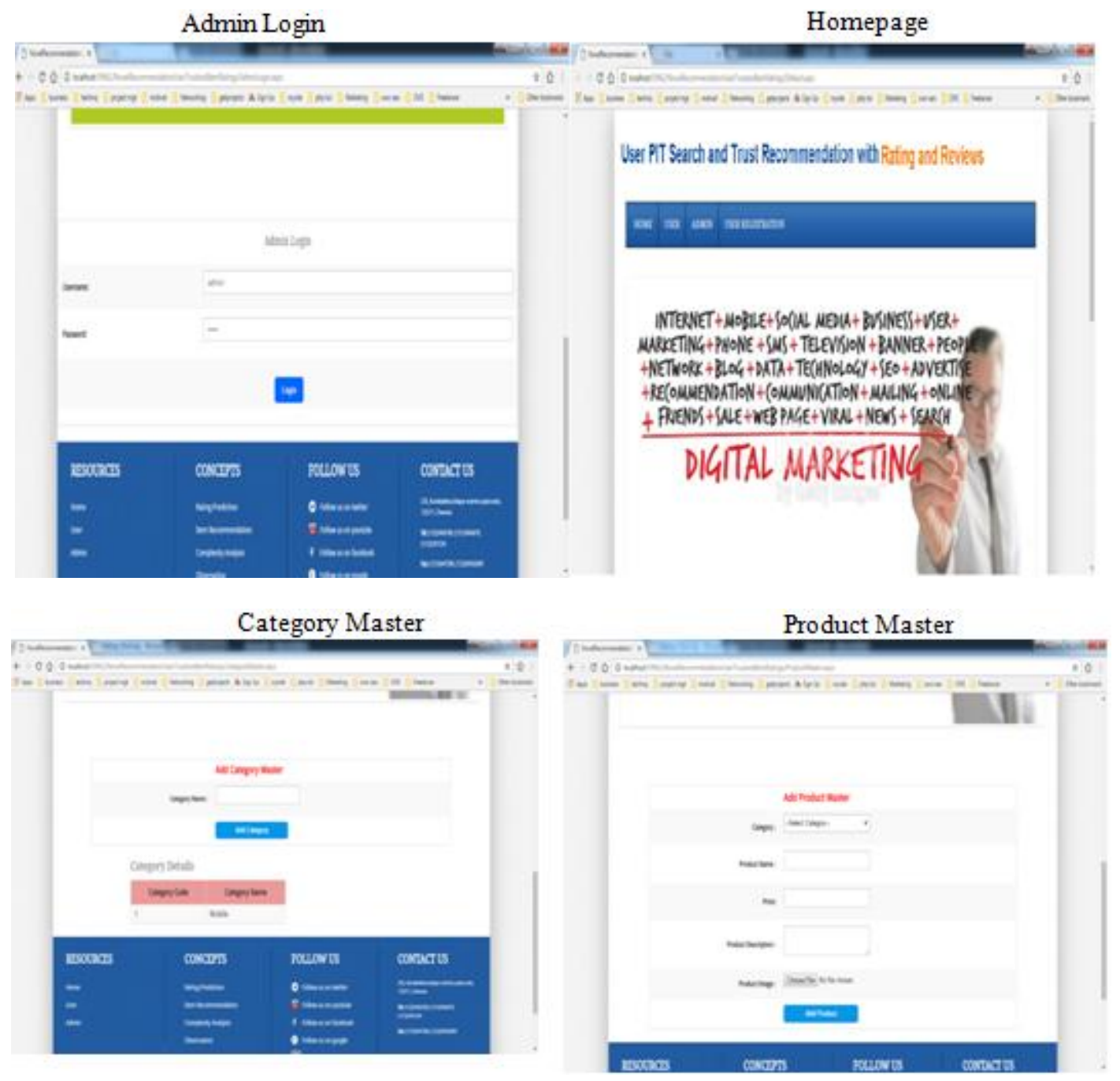

Volume 6 Issue 7, July 2017

www.ijsr.net

Licensed Under Creative Commons Attribution CC BY 


\section{International Journal of Science and Research (IJSR) \\ ISSN (Online): 2319-7064}

Index Copernicus Value (2015): 78.96 | Impact Factor (2015): 6.391

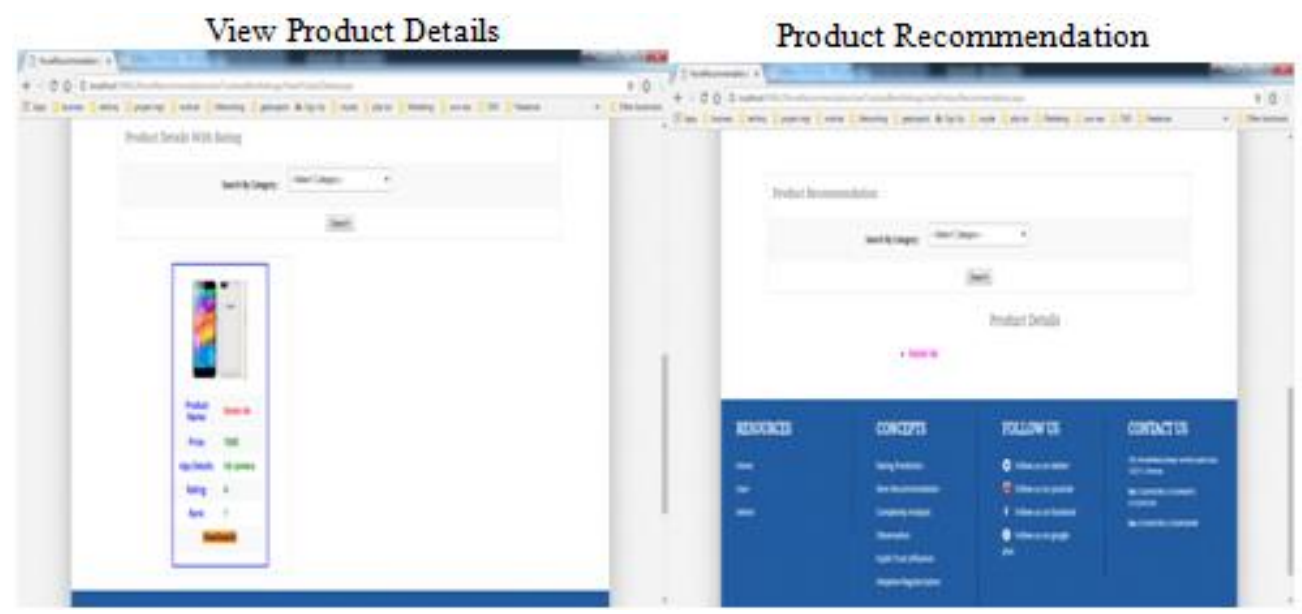

\section{Conclusion}

A novel trust-based matrix factorization model which incorporated both rating and trust information. Our analysis of trust in four real-world data sets indicated that trust and ratings were complementary to each other, and both pivotal for more accurate recommendations. With the rapid growth of online social networks, the social based recommender systems have become more and more popular and important. In this work, we focused on the social item recommendation problem in the implicit feedback and proposed a novel social item ranking method called Social $\mathrm{BPR}_{\mathrm{CRWR}}$. We derived the optimization criterion of Social $\mathrm{BPR}_{\mathrm{CRWR}}$ from a Bayesian analysis of the problem, where we introduced the social trust interactions among users from the theory of social influence to improve the performance of item recommendation. To understand the true social trust relations, we further proposed a category-sensitive random walk method CRWR to estimate the multi-faceted trust strengths.

\section{Future Enhancement}

This work suggests several interesting directions for future work. We want to extend the model to handle negative trust relations, since some social networks allow users to express distrust towards other users. In this paper, we performed experiments on different ranges of $\lambda_{1}$ As future work, we investigate the possibility of tuning $\lambda_{r}$ automatically. Finally, cold start items have not been addressed in this paper, and it should be explored how the model can be extended so that the feature vectors of cold start items are also learnt efficiently.

\section{References}

[1] Y. Huang, X. Chen, J. Zhang, D. Zeng, D. Zhang, and X. Ding, "Single-trial fERPsgdenoising via collaborative filtering on fERPsg images," Neurocomputing, vol. 149, Part B, pp. 914 - 923, 2015.

[2] X. Luo, Z. Ming, Z. You, S. Li, Y. Xia, and H. Leung, "Improving network topology-based protein interactome mapping via collaborative filtering," Knowledge-Based Systems (KBS), vol. 90, pp. 23-32, 2015.

[3] G. Guo, J. Zhang, and D. Thalmann, "A simple but effective method to incorporate trusted neighbors in recommender systems," in Proceedings of the 20th
International Conference on User Modeling, Adaptation and Personalization (UMAP), 2012, pp. 114- 125.

[4] H. Ma, H. Yang, M. Lyu, and I. King, "SoRec: social recommendation using probabilistic matrix factorization," in Proceedings of the 31st International ACM SIGIR Conference on Research and Development in Information Retrieval (SIGIR), 2008, pp. 931-940.

[5] M. Jamali and M. Ester, "A matrix factorization technique with trust propagation for recommendation in social networks," in Proceedings of the 4th ACM Conference on Recommender Systems (RecSys), 2010, pp. 135-142.

[6] B. Yang, Y. Lei, D. Liu, and J. Liu, "Social collaborative filtering by trust," in Proceedings of the 23rd International Joint Conference on Artificial Intelligence (IJCAI), 2013, pp. 2747-2753.

[7] H. Fang, Y. Bao, and J. Zhang, "Leveraging decomposed trust in probabilistic matrix factorization for effective recommendation," in Proceedings of the 28th AAAI Conference on Artificial Intelligence (AAAI), 2014, pp. 30-36.

[8] G. Guo, J. Zhang, and N. Yorke-Smith, "TrustSVD: collaborative filtering with both the explicit and implicit influence of user trust and of item ratings," in Proceedings of the 29th AAAI Conference on Artificial Intelligence (AAAI), 2015, pp. 123-129.

[9] W. Yuan, D. Guan, Y. Lee, S. Lee, and S. Hur, "Improved trust aware recommender system using small-worldness of trust networks," Knowledge-Based Systems, vol. 23, no. 3, pp. 232-238, 2010. 